\title{
Robust design of microlenses arrays employing dielectric resonators metasurfaces
}

\author{
Fabrizio Silvestria $^{\mathrm{a}, \mathrm{b}}$, Giampiero Gerini ${ }^{\mathrm{a}, \mathrm{b}}$, and Stefan M.B. Bäumer ${ }^{\mathrm{a}}$ \\ ${ }^{a}$ TNO, Optics Department, Stieltjesweg 1, 2628CK Delft, The Netherlands \\ ${ }^{\mathrm{b}}$ Eindhoven University of Technology, Postbus 513, 5600MB Eindhoven, The Netherlands
}

\begin{abstract}
In the last years, much interest has grown around the concept of optical surfaces employing high contrast dielectric resonators. However, a systematic approach for the design of this optical surfaces under particular requirements has never been proposed. In this contribution, we describe this approach applied to the robust design of an array of microlenses characterized by a numerical aperture of NA=0.19 with a field of view of FOV $= \pm 60$ mrad in a bandwidth of $20 \mathrm{~nm}$. Typically, dielectric resonators are engineered in such a way to have almost full transmissive surfaces with locally tunable phase. However, considering the multiple wavelengths and angles under which the lenses may work, it is difficult to get uniform transmission characteristics for all the dielectric resonators employed. The design strategy, here proposed, uses a particle swarm optimization routine to find the best resonator distribution able to meet the requirements considering the amplitude and phase dispersive characteristics of the resonators surfaces. In the optimization process, also the effects of possible manufacturing inaccuracies, such as variations of resonators radii, are taken into account, allowing a robust design of the structure, within the given manufacturing tolerances. Different designs, operating at $405 \mathrm{~nm}$ and $635 \mathrm{~nm}$, are presented and their performances are discussed.
\end{abstract}

Keywords: Metasurfaces, diffractive lens, optical design

\section{INTRODUCTION}

Metasurface optical components constitute an emerging field of research. The term optical metasurfaces mainly refer to a collection of optical subwavelength scatterers, which, by means of their topology, electric and geometric characteristics, can manipulate light as conventional refractive/reflective components do. With their reduced form factor, metasurfaces are an attractive solution for several optical functionalities, like polarization manipulation, phase tunable surfaces, field enhancement. A limited collection of these works can be found in. ${ }^{1-16}$ In the case of flat lenses, metasurface have been usually designed by mapping the required phase distribution onto a function of the characteristic parameters of the basic scattering elements, e.g. sizes of the dielectric resonators, ${ }^{1,5,7,10,11}$ orientation of the dielectric posts, ${ }^{16}$ local period of plasmonic gratings. ${ }^{13}$ The desired phase distributions are derived by means of analytic/numerical techniques, based on the knowledge of the incoming and desired outgoing wavefront at the lens surface. However, it is known that diffractive optical elements, such as metasurfaces, suffer from chromatic aberrations. ${ }^{17}$ Moreover, in a typical optical design, also the performance under different angles of incidence (field of view, FOV) is of interest. Up to now, although they have been valuable attempts of tackling the metasurface design problem from a more practical point of view, ${ }^{18}$ a systematic approach to design optical components, especially flat lenses, employing metasurfaces, is not available. In this contribution, we propose a way to handle all optical requirements (encircled energy, bandwidth - BW, field of view - FOV, geometric requirements) in the design phase, in order to define the optimal metasurface. Moreover, the proposed approach takes into account also the sensitivity of the lens under variations of the optical characteristics, due to manufacturing errors. This is achieved by means of a robust optimization strategy.

This manuscript is organized as follows: section 2 deals with the design strategy and the description of the metasurface implementation; in section 3, two application examples (two optically equivalent lenses working respectively at $405 \mathrm{~nm}$ and $635 \mathrm{~nm}$ ) are presented; the final section 4 concludes the contribution.

Further author information: (Send correspondence to F.S.)

F.S.: E-mail: fabrizio.silvestri@tno.nl, Telephone: +31 888665935

High Contrast Metastructures VI, edited by Connie J. Chang-Hasnain, Andrei Faraon,

Fumio Koyama, Weimin Zhou, Proc. of SPIE Vol. 10113, 101130M · @ 2017 SPIE

CCC code: $0277-786 \mathrm{X} / 17 / \$ 18 \cdot$ doi: $10.1117 / 12.2250477$

Proc. of SPIE Vol. 10113 101130M-1 


\section{ROBUST DESIGN STRATEGY}

Refractive and reflective conventional components are in general designed with the help of computer-assisted design (CAD) softwares, ${ }^{19}$ such as CODE V ${ }^{20}$ or Zemax OpticStudio. ${ }^{21}$ These software packages compute the optical performances by tracing rays through the group of lenses constituing the optical system. The designer defines what are the design variables (e.g. size of apertures, surfaces' types, surfaces' curvatures, distances, etc), some additional input data (e.g. refractive indices, conditions of operations, etc) and a merit function that should include all the performance requirements. By iteratively tracing rays, computing the merit function and applying optimization techniques, these softwares help the designer in the not simple task of designing the optical system. Whereas also diffractive optical surfaces, based on quantized refractive profiles ${ }^{17}$ can still be analyzed and optimized with ray tracinq softwares, this is not currently done with metasurface lenses. For this reason, tools useful to design metasurface optical components are highly sought. However, with metasurface optical elements, an approach based on physical optics appears to be more suited than ray-tracing. ${ }^{1,18}$ To reach this objective, the possibility of integrating all the optical requirements in a unique cost function and exploiting optimization techniques to achieve an optimal design, is definitely needed. For this reason, we have developed a robust design strategy that exploits a combined full wave - scalar diffraction theory (SDT) tool for the analysis and a particle swarm optimization ${ }^{22}$ to end up with an optimal metasurface in order to satisfy given requirements. The basic features of the proposed strategy are:

- angular and wavelength possible dispersive effects of the metasurfaces are taken into account in the design phase of the optical component;

- unwanted apodization effects are taken into account during the design phase. These effects are due to the not uniform transmission distributions;

- decoupling of the computationally heavy full wave simulations of the metasurfaces and the optimization of the optical component. The optimization is not carried out on the local scatterers, but in a global sense;

- the effects of manufacturing imperfections are considered already in the design phase.

This procedure needs as input the electromagnetic transmission characteristics of the elementary constituents of the metasurface (resonators, gratings, slots, etc). For this work, we have adopted arrays of dielectric cylindrical resonators, embedded in a low contrast material. By acting only on the radii of the resonators, it is possible to obtain transmission profiles characterized by a high transmission and a phase tunable in the complete $2 \pi$ range. Two different arrays have been studied: one (MS1) with Gallium Nitride (GaN, n=2.55 @ $405 \mathrm{~nm}$ ) resonators embedded in fused Silica $\left(\mathrm{SiO}_{2}, \mathrm{n}=1.47 @ 405 \mathrm{~nm}\right)$ and one (MS2) with Silicon ( $\mathrm{Si}, \mathrm{n}=3.87-\mathrm{j} 0.00085$ @ $635 \mathrm{~nm})$ resonators embedded in fused Silica $\left(\mathrm{SiO}_{2}, \mathrm{n}=1.46 @ 635 \mathrm{~nm}\right)$. The two solutions obviously work at different wavelength ranges, due to the resonant, narrowband behavior of the resonators. Table 1 reports all the characteristics of the resonators used for the two designs. The transmission characteristics have been computed with an infinite periodic array assumption, with the commercial finite element software Comsol Multiphysics. ${ }^{23}$ Several computations have been performed for different wavelengths and different angles of incidence. The transmission profiles for the two structures are reported in figure 1.

Table 1. Characteristic parameters dielectric resonators metasurfaces

\begin{tabular}{|c|c|c|}
\hline & Metasurface MS1 @ 405 nm & Metasurface MS2 @ 635 nm \\
\hline Resonators material & Gallium Nitride $(\mathrm{GaN}) \mathrm{n}=2.55$ & Silicon $(\mathrm{Si}) \mathrm{n}=3.87-\mathrm{j} 8.5 \cdot 10^{-4}$ \\
\hline Host material & Fused Silica $\left(\mathrm{SiO}_{2}\right) \mathrm{n}=1.47$ & Fused Silica $\left(\mathrm{SiO}_{2}\right) \mathrm{n}=1.46$ \\
\hline Thickness host material & $394 \mathrm{~nm}\left(+500 \mu \mathrm{m}\right.$ back $\mathrm{SiO}_{2}$ layer $)$ & $665 \mathrm{~nm}\left(+500 \mu \mathrm{m}\right.$ back $\mathrm{SiO}_{2}$ layer $)$ \\
\hline Lattice period & $220 \mathrm{~nm}$ & $377 \mathrm{~nm}$ \\
\hline Height resonators & $100 \mathrm{~nm}$ & $96 \mathrm{~nm}$ \\
\hline Error variance $\sigma^{2}$ & $5 \mathrm{~nm}$ & $5 \mathrm{~nm}$ \\
\hline
\end{tabular}




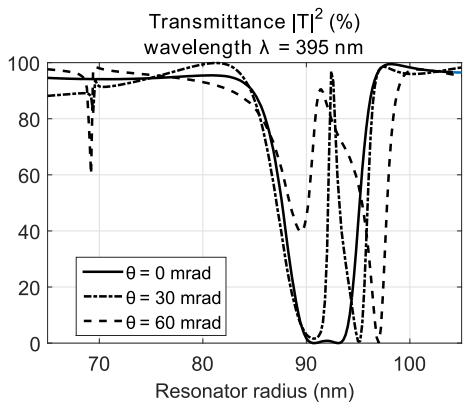

(a)

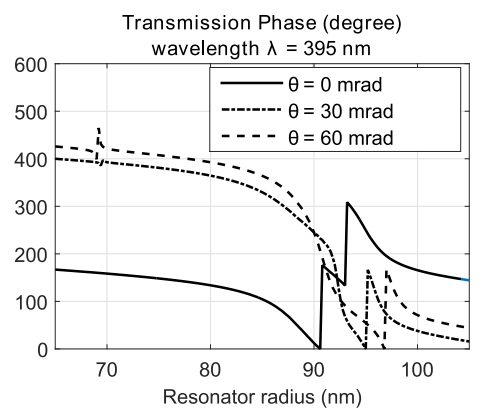

(d)

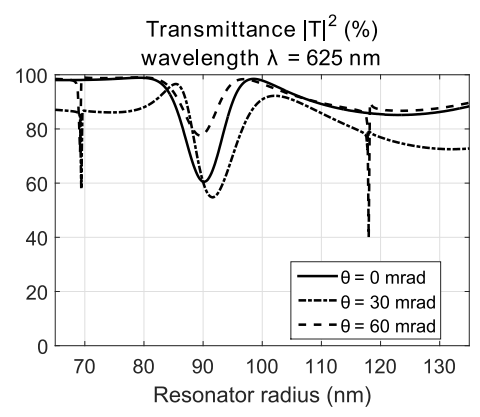

(g)

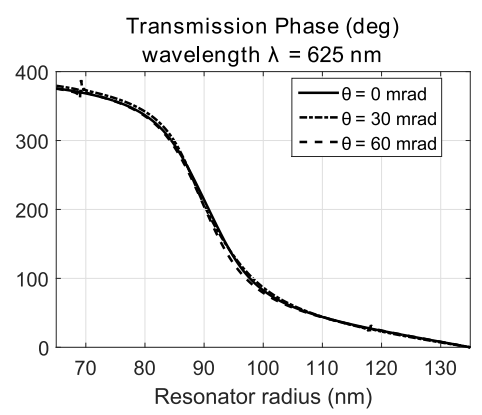

(j)

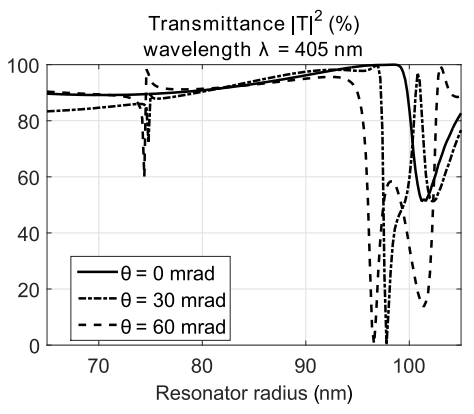

(b)

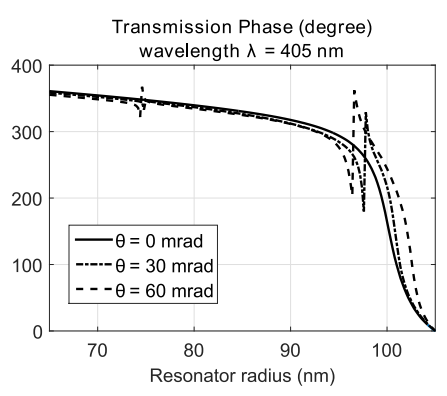

(e)

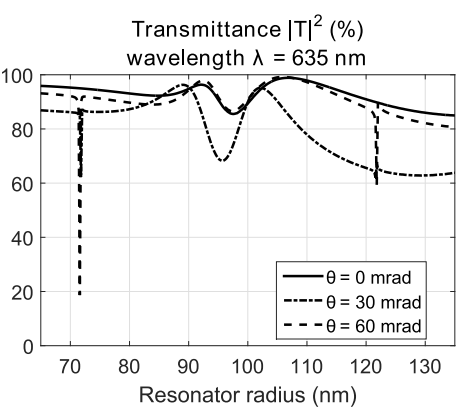

(h)

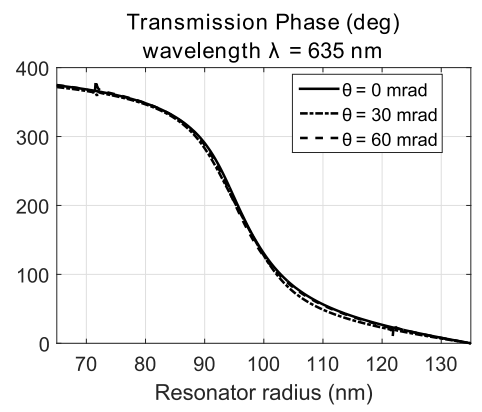

$(\mathrm{k})$

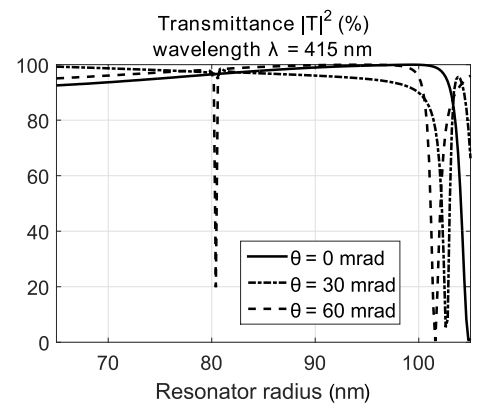

(c)

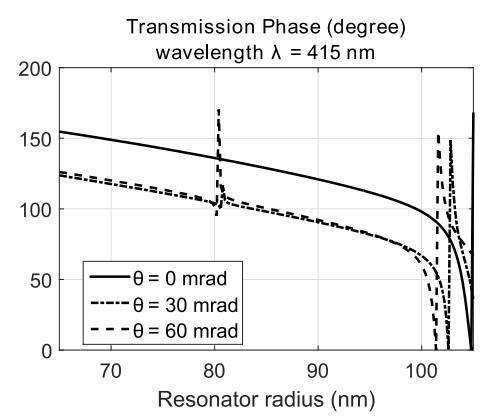

(f)

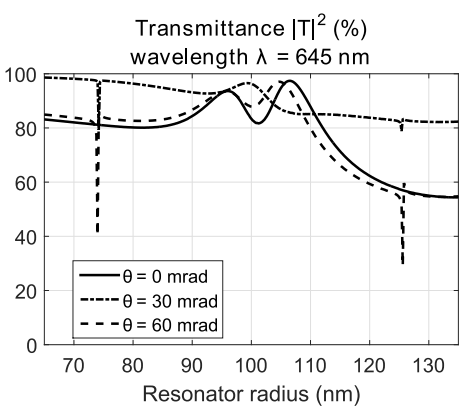

(i)

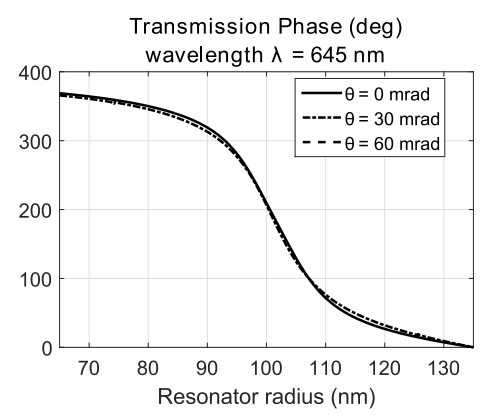

(l)

Figure 1. Transmission characteristics of the array of resonators vs. resonators radius, for different wavelengths and angles of incidence: (a,b,c) transmittance of MS1, (d,e,f) transmission coefficient phase of MS1, $(\mathrm{g}, \mathrm{h}, \mathrm{i})$ transmittance of MS2, $(\mathrm{j}, \mathrm{k}, \mathrm{l})$ transmission coefficient phase of MS2

In addition to the transmission characteristics, also all the other requirements must be considered in the 
optimization process. These can be expressed as target intensity distributions at the image plane. A target distribution should be defined for each different condition of operation. For the design of the lenses, as a target distribution, the Airy's pattern is used as representative of an optical system free of aberrations. A flowchart of the procedure is reported in figure 2 and in the algorithm ?? description. The optimization variables are the curvature $C$, the conic constant $K$ and the polynomiaml coefficients $C_{i}$ of a circular symmetric phase profile defined as

$$
\phi(r)=\frac{2 \pi}{\lambda_{0}}\left(\frac{C r^{2}}{1+\left[1-(1+K) C^{2} r^{2}\right]^{1 / 2}}+\sum_{i=1}^{6} C_{i} r^{2(i+1)}\right) .
$$

Such a general profile avoids a computationally heavy local topology optimization and allows acting directly on the best profile of the optical phase surface. The operations performed at each iteration, for each particle in the PSO algorithm, are reported in the algorithm ?? description.

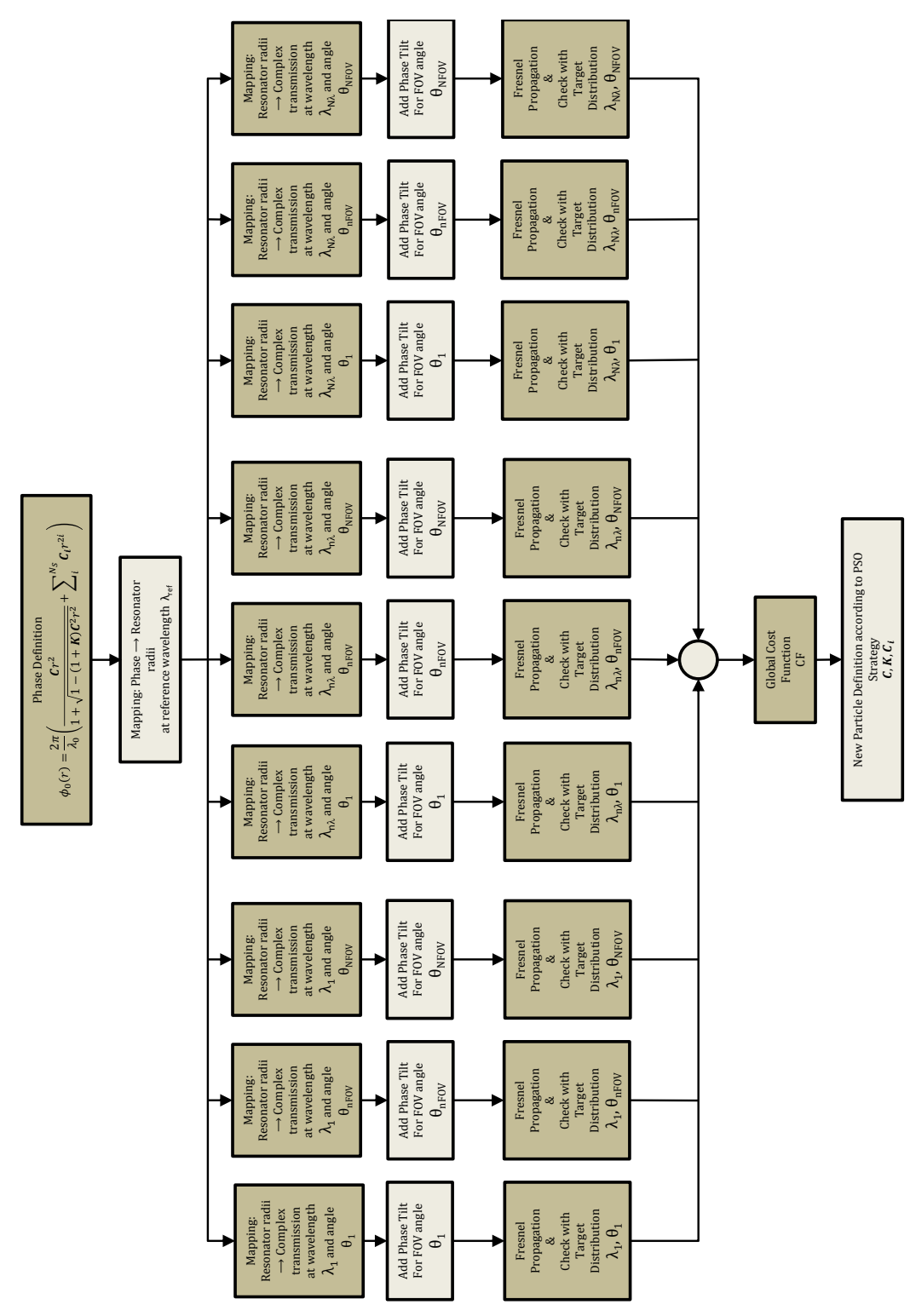

Figure 2. Flowchart of the optimization strategy. 


\section{Algorithm 1: Optimization procedure steps for the design of metasurface lenses}

1. Computation of the phase distribution $\phi(r)$ for the new set of optimization variables $C, K, C_{i}$.

2. Mapping of the phase distribution into a physical distribution of resonators exploiting the curves of figure 1 , at the reference wavelength $\lambda_{0}$ (in this work the reference wavelength has been set equal to the central wavelength of the BW).

3. Mapping of the nominal resonators distribution into the complex transmission function for different wavelengths, $\lambda_{n_{\lambda}}$, and angles of incidence, $\theta_{n_{F O V}}$, according to the complex transmission vs. resonators curves (figure 1). This additional mapping provides the performances of the chosen distribution of resonators at the given iteration, considering their dispersive behavior.

\section{Addition of a linear phase slope for tilted incidence.}

5. Propagation of the optical beam up to the given image plane. Different propagation techniques may be used. ${ }^{17,24}$ For this work, we have used a numerical implementation of the Fresnel propagation exploiting the Fast Fourier Transform (FFT). By defining as $T\left(x_{o}, y_{o} ; n_{\lambda}, n_{F O V}\right)$ the complex transmission distribution at the metasurface plane, the complex distribution at the image plane is computed by

$$
I\left(x_{i}, y_{i} ; n_{\lambda}, n_{F O V}\right)=\left|\iint_{-\infty}^{\infty} T\left(x_{o}, y_{o} ; n_{\lambda}, n_{F O V}\right) e^{-i k \sin \theta_{n_{F O V}} x} e^{i \frac{k}{2 z_{i}}\left(x_{o}^{2}+y_{o}^{2}\right)} e^{-i \frac{k}{z_{i}}\left(x_{i} x_{o}+y_{i} y_{o}\right)} d x_{o} d y_{o}\right|^{2}
$$

where $k$ is the wavenumber, $z_{i}$ the distance between the metasurface and the image plane, and $n_{\lambda}, n_{F O V}$ are two indices indicating the wavelengths and angles of incidence, respectively. As a consequence of the use of the FFT, a fine sampling period at the metasurface plane (equal to the lattice period of the metasurface) results in a coarse period at the image plane. In fact, for Fresnel propagation, these two quantities are related by

$$
\delta_{i}=\frac{\lambda z_{i}}{N \delta_{o}}
$$

where $\delta_{i}, \delta_{o}$ are the sampling periods at the image and metasurface plane respectively, $N$ the number of points used in the grid and $\lambda$ the wavelength. For this reason, an interpolation through spline curves may be beneficial, at the image plane, to get smoother curves.

6. Evaluation of the cost function. This should reflect the desired requirements. For this work, a pointwise comparison between the actual image intensity distribution and an ideal one, namely an Airy's disc distribution $I_{\text {Airy }}\left(x ; n_{\lambda}, n_{F O V}\right)$ located at the Gaussian Image Point (GIP), $x_{G I P}\left(n_{\lambda}, n_{F O V}\right)$, has been used. All the values of the cost functions, obtained by the comparisons at different wavelengths and angles of incidence, are combined in a global cost function defined as

$$
C F=\sum_{n_{\lambda}=1}^{N_{\lambda}} \sum_{n_{F O V}=1}^{N_{F O V}}\left\|\left[x_{i}-x_{i}^{G I P}\left(n_{F O V}\right)\right]^{8}\left[I\left(x_{i}, 0 ; n_{\lambda}, n_{F O V}\right)-I_{A i r y}\left(x_{i}, 0 ; n_{\lambda}, n_{F O V}\right)\right]\right\|^{2} .
$$

The eighth power of the distance weights the point-wise error in intensity in such a way that errors far from the GIP are weighted more. This helps in preventing the final image distribution to be characterized by ripples containing high amounts of energy far from the GIP, affecting consequently the final focusing performances of the lens.

7. Ranking of each particle according to the PSO and generation of the new solution. In principle, here any optimization strategy could be used. The choiche of PSO has been dictated by the fact that it can handle continuous variables and, differently from gradient-based optimization techniques, does not suffer from the problem of local optima. 
The strategy proposed, owing to its modularity, can be used independently from the model used for the metasurface and the propagation. For example, if the metasurface is modeled as a surface impedance or admittance function ${ }^{25}$ instead of as a complex transmission function, the same strategy can be used to find the optimal surface impedance/admittance distribution for given requirements and conditions of operations. The strategy, as it is presented in figure 2, does not take into account any effect of manufacturing tolerances. These can severally affect the final performances of the metasurface lens, especially for those devices working at smaller wavelengths, where the lithographic tolerances are in percentage larger. To prevent this, the proposed strategy is modified accordingly to the robust optimization principles. ${ }^{22}$ For each point in the optimization landscape, several cost functions are computed for samples around the nominal point within a given region. The final cost function for the nominal point is found by averaging the single cost functions. This can be interpreted as a Monte-Carlo evaluation of the cost function. The region, within which the additional parameters points are selected, is delimited accordingly to the maximum expected deviation. In the case of dielectric resonators metasurfaces, the manufacturing errors that mainly affects the performances are errors in the radii of the resonators. Indeed, it can be seen from the phase curves, figure 1, that even a small error in the resonators radius, in the order of less than $10 \mathrm{~nm}$, can result in a local wavefront error of tens of degrees. Instead of applying a variation in the optimization variables $C, K, C_{i}$, which are global variables, and therefore cannot directly represent a local error, the robust optimization paradigm has been applied in a slightly different way from what reported in. ${ }^{22}$ This is described by the flowchart of figure 3, that must be interpreted as an addition to the main flowchart of figure 2 .

In order to model local radius errors, once a nominal resonator distribution has been found (step 3 in algorithm 1), $N_{M C}$ samples are generated, adding, on top of the nominal dimensions, variations of radius extracted from a normal distribution $\mathcal{N}\left(0, \sigma^{2}\right)$. For each resonators distribution, the optical beam is propagated considering all the different conditions of operation (steps 4-6). The average of all cost functions for each sample is the Monte Carlo cost function for the given particle. In this work, $N_{M C}=10$ samples have been taken for each of the 20 particles of the PSO algorithm. The variance for the normal distribution has been set to $\sigma^{2}=5 \mathrm{~nm}$.

\section{DESIGN OF FLAT LENSES WITH EXTENDED BANDWIDTH AND FIELD OF VIEW}

The procedure described in section 2 has been applied to the design of two flat lenses. The geometrical and optical features of the lenses are summarized in table 2. To evaluate the performances of the designed lenses,

Table 2. Characteristic parameters of the designed metasurface lenses

\begin{tabular}{|c|c|}
\hline Accepted beam diameter & $600 \mu \mathrm{m}$ \\
\hline Focal length & $1.5 \mathrm{~mm}$ \\
\hline Numerical aperture (image space) & 0.196 \\
\hline Telecentricity & Only in the image space \\
\hline BW & $20 \mathrm{~nm}$ \\
\hline FOV & $\pm 60 \mathrm{mrad}$ \\
\hline
\end{tabular}

the point spread functions (PSFs) obtained for different conditions of operation have been compared with the ideal ones. Moreover, the encircled energy has been computed and plotted towards the wavelength and the angle of incidence. These curves are reported in figure 4, for the lens realized with MS1, and in figure 5, for the lens realized with MS2.

The PSFs shown are normalized with respect to their peak value. This is done to discard the effects of the average transmission loss due to the dips presents in the resonators characteristics (figure 1), which are quite severe, especially for the lens employing MS1. However, for lithographic writing applications, where laser or light-emitting diodes (LEDs) sources are used, reflection losses in the beam are not a particular issue. From the comparison of the PSFs it can be seen that the two lenses ensure a central spot size close to the ideal one, even for large field points $(\theta=60 \mathrm{mrad})$. However, ripples far from the GIP are present in the PSFs and these decrease the performances of the lens in terms of encircled energy. The obtained values for this last figure of merit are 


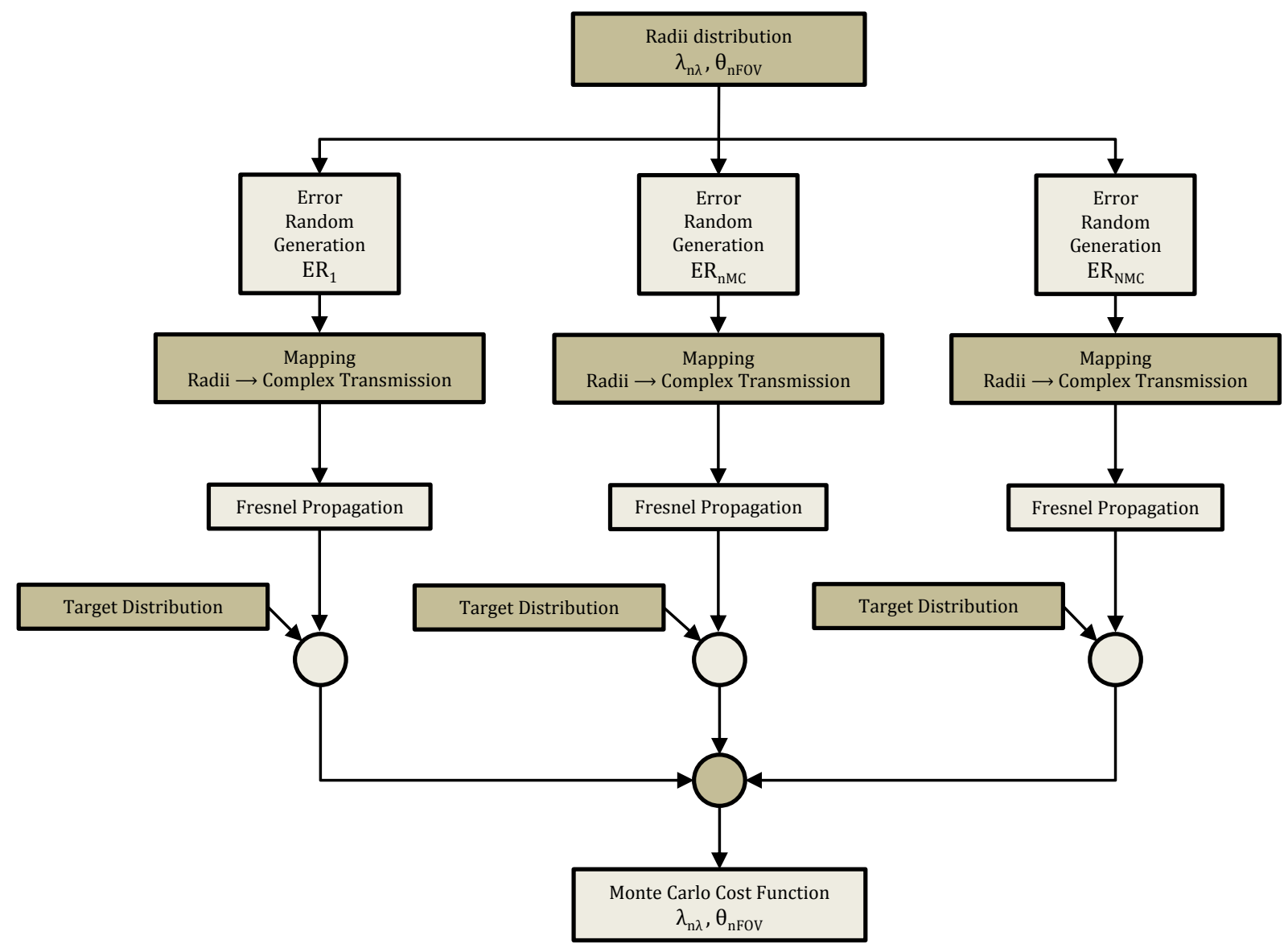

Figure 3. Flowchart for the robust extension of the flowchart of figure 2. The above steps are intended to replace the Fresnel Propagation \& Check with Target Distribution block present in the original flowchart.

quite far from the ideal value of $68 \%$ (lens free from aberration), in the case of the lens realized with MS1. For MS2, at least at the central wavelength, the encircled energy value is close to the ideal one for on-axis excitation. The large difference from the ideal performances is not ascribable to the design procedure, but more to the tight requirements chosen for the two examples. A deeper discussion about this can be found in the paper. ${ }^{26}$

It is interesting to compare these results with the ones obtained when a theoretical phase profile, although optimized, is directly mapped on a resonators distribution, without considering their dispersive behavior and the intrinsic apodization given by the non uniform transmission distribution. For both designs, a final Monte Carlo simulation, based on 50 samples each, has been carried out, to check the deviation from nominal performances, when errors are present in the resonators shapes. Looking at the encircled energy for the MS1, figure 6, two main conclusions can be taken:

- by taking into account the wavelength and angular dispersive behavior of the resonators, the robust optimization design procedure is able to find a design with improved performances at the edges of the BW (unfortunately, this improvement pays off in terms of a decrease in performances, at the central wavelength);

- by applying the robust optimization paradigm with the computation of the Monte-Carlo simulations in the design phase, the design procedure is able to find a design less sensitive to manufacturing tolerances 


\section{DISCUSSION AND CONCLUSIONS}

In this paper, a robust optimization strategy for the design of metasurface lenses has been proposed. This procedure combines full-wave simulations and scalar diffraction theory algorithms to evaluate the performance of each lens, under different conditions of operations (wavelength, angle of incidence). Successively, this analysis is used in an optimization loop. Besides taking into account possible dispersive effects of the metasurface, the optimization strategy exploits a Monte Carlo strategy to take into account manufacturing deviations in the final optical performance of the designed component. The proposed strategy is general and can be customized for different physical implementations of the metasurface (dielectric scatterers, metallic scatterers, slots on metallic planes, etc.), different models for the metasurface-incident light interaction (complex transmission distributions, surface impedance/admittance distributions, etc.) and for different functionalities and requirements (lenses, deflectors, polarizers). The procedure has been applied to the design of two telecentric lenses working in the violet $(395 \mathrm{~nm}-415 \mathrm{~nm})$ and red $(625 \mathrm{~nm}-645 \mathrm{~nm})$ visible bands. The lenses are designed using high contrast dielectric resonator metasurfaces. The results show clearly an improvement in performances, at the extremes of BW and FOV, compared to designs obtained by optimizing the metasurface behavior only at the central wavelength. Moreover, the procedure succeeds in finding optical designs more robust against manufacturing imperfections, as demonstated by the reduced sensitivity to manufacturing tolerances.

\section{REFERENCES}

[1] Arbabi, A., Horie, Y., Bagheri, M., and Faraon, A., "Subwavelength-thick lenses with high numerical apertures and large efficiency based on high-contrast transmitarrays," Nature Communication 6, 7069 (2015).

[2] West, P. R., Stewart, J. L., Kildishev, A. V., Shalaev, V. M., Shkunov, V. V., Strohkendl, F., Zakharenkov, Y. A., Dodds, R. K., and Byren, R., "All-dielectric subwavelength metasurface focusing lens," Optics Express 22, 26212-26221 (2014).

[3] Vo, S., Fattal, D., Sorin, W. V., Peng, Z., Tran, T., and Beausoleil, R. G., "Sub-wavelength grating lenses with a twist," IEEE Photonics Technology Letters 26, 1375-1378 (2014).

[4] Decker, M., Staude, I., Falkner, M., Dominguez, J., Neshev, D. N., Brener, I., Pertsch, T., and Kivshar, Y. S., "High-efficiency dielectric huygens' surfaces," Advanced Optical Materials 3, 813-820 (2015).

[5] Silvestri, F., Bernal Arango, F., Vendel, K. J. A., Gerini, G., Bäumer, S. M. B., and Koenderink, A. F., "Optical antennas for far and near field metrology," Proc. 10th European Conference on Antennas and Propagation, 1-4 (2016).

[6] Zhan, A., Colburn, S., Trivedi, R., Fryett, T. K., Dodson, C. M., and Majumdar, A., "Low-contrast dielectric metasurface optics," ACS Photonics 3, 209-214 (2016).

[7] Zou, L., Withayachumnankul, W., Shah, C. M., Mitchell, A., Bhaskaran, M., Sriram, S., and Fumeaux, C., "Dielectric resonator nanoantennas at visible frequencies," Optics Express 21, 1344-1352 (2013).

[8] Arbabi, A., Horie, Y., Bagheri, M., and Faraon, A., "Dielectric metasurfaces for complete control of phase and polarization with subwavelength spatial resolution and high transmission," Nature Nanotechnology 10, 937-943 (2015).

[9] Silvestri, F., Pisano, E., Gerini, G., Lancellotti, V., and Galdi, V., "Nanoresonator based dielectric surfaces for light manipulation," Proc. of European Microwave Conference EuMC 2015, Paris 2015, 1240-1243 (2015).

[10] Aieta, F., Kats, M. A., Genevet, P., and Capasso, F., "Multiwavelength achromatic metasurfaces by dispersive phase compensation," Science 347, 1342-1345 (2015).

[11] Arbabi, E., Arbabi, A., Kamali, S. M., Horie, Y., and Faraon, A., "Multiwavelength polarization-insensitive lenses based on dielectric metasurfaces with meta-molecules," Optica 3, 628-633 (2016).

[12] Deng, Z. L., Zhang, S., and Wang, G. P., "A facile grating approach towards broadband, wide-angle and high efficiency holographic metasurfaces," Nanoscale 8, 1588-1594 (2016).

[13] Deng, Z. L., Zhang, S., and Wang, G. P., "Wide-angled off-axis achromatic metasurfaces for visible light," Optics Express 24, 23118-23128 (2016). 
[14] Headland, D., Carrasco, E., Nirantar, S., Withayachumnankul, W., Gutruf, P., Schwarz, J., Abbott, D., Bhaskaran, M., Sriram, S., Perruisseau-Carrier, J., and Fumeaux, C., "Dielectric resonator reflectarray as high-efficiency nonuniform terahertz metasurface," ACS Photonics 3, 1019-1026 (2016).

[15] Jahani, S. and Jacob, Z., "All-dielectric metamaterials," Nature Nanotechnology 11 (2015).

[16] Khorasaninejad, M., Chen, W. T., Devlin, R. C., Oh, J., Zhu, A., and Capasso, F., "Metalenses at visible wavelengths: Diffraction-limited focusing and subwavelength resolution imaging," Science 352, 1190 (2016).

[17] Kress, B. C. and Meyrueis, P., [Applied Digital Optics], John Wiley and Sons (2009).

[18] Byrnes, S. J., Lenef, A., Aieta, F., and Capasso, F., "Designing large, high-efficiency, high-numerical aperture, transmissive meta-lenses for visible light," Optics Express 24, 5110 (2016).

[19] Smith, W. J., [Modern Optical Engineering], McGraw Hill Inc. (1990).

[20] "Synopsis CODE V." 2016 Synopsis.

[21] "Zemax OpticStudio." 2016 Zemax LLC.

[22] Dippel, C. E. J., "Using particle swarm optimization for finding robust optima." http://natcomp.liacs . nl/code/RO_PSO/rpso_report.pdf. (Accessed: 28 November 2016).

[23] "COMSOL Multiphysics 5.0." 2016 Comsol Inc.

[24] Schmidt, J. D., [Numerical simulation of optical wave propagation], SPIE Press (2010).

[25] Estakhri, N. M. and Alù, A., "Recent progress in gradient metasurfaces," J. Opt. Soc. Am. B 33, A21-A30 (Feb 2016).

[26] Silvestri, F., Gerini, G., Bäumer, S. M. B., and van Zwet, E. J., "Robust design procedure for dielectric resonator metasurface lens array," Optics Express 24, 29153-29169 (2016). 


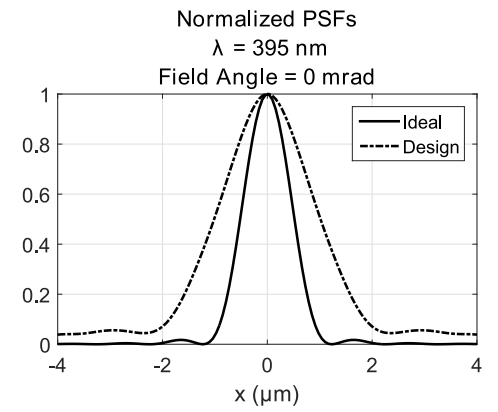

(a)

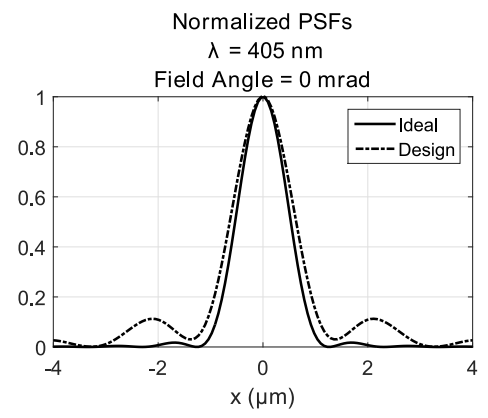

(d)

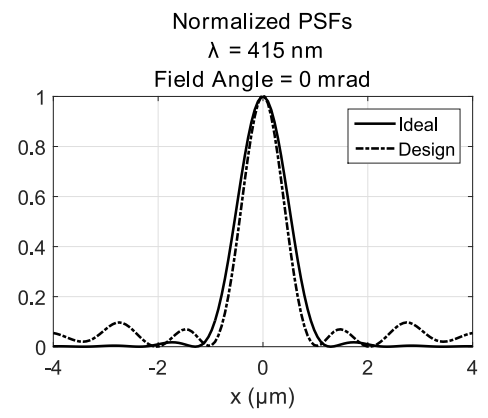

(g)

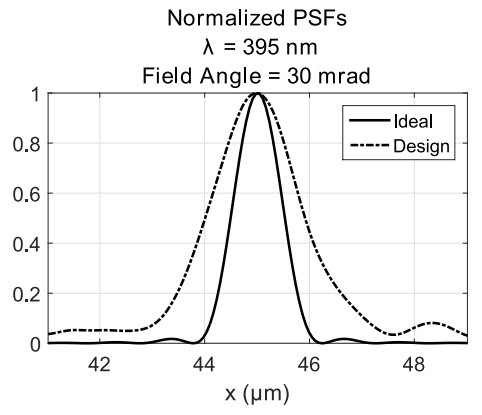

(b)

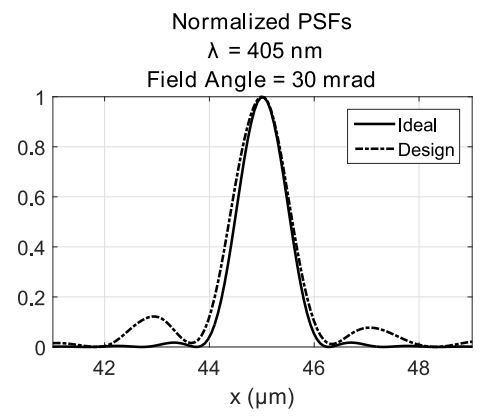

(e)

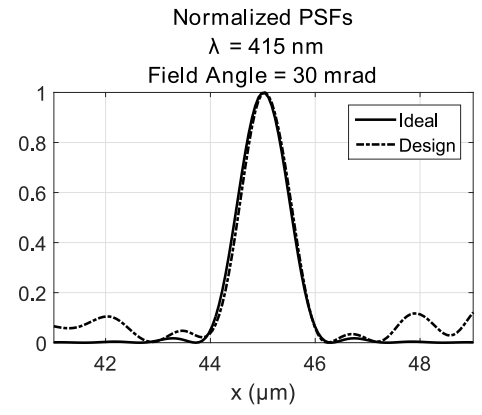

(h)

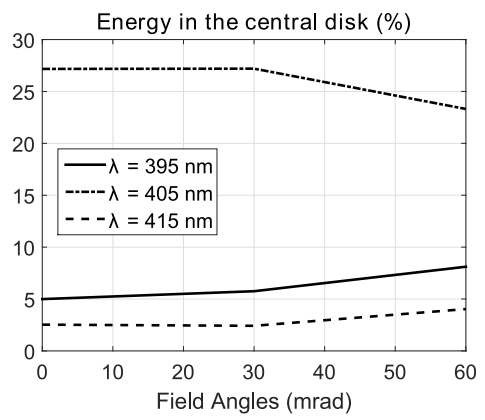

(j)

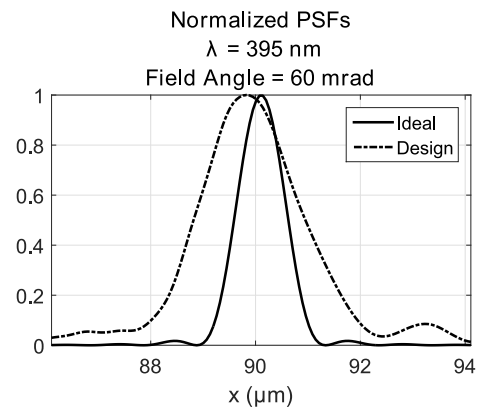

(c)

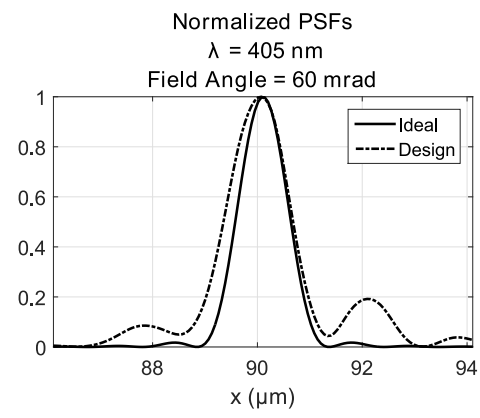

(f)

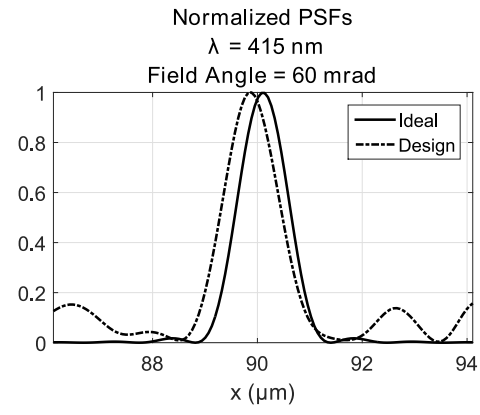

(i)

Figure 4. Focal plane intensity distributions for different wavelengths and angles of FOV for the dielectric resonator metasurface lens realized with MS1: (a,b,c) wavelength $\lambda=395 \mathrm{~nm}, \mathrm{FOV}=0,30,60 \mathrm{mrad}$; (d,e,f) wavelength $\lambda=405 \mathrm{~nm}$, FOV $=0,30,60 \mathrm{mrad} ;(\mathrm{g}, \mathrm{h}, \mathrm{i})$ wavelength $\lambda=415 \mathrm{~nm}$, FOV $=0,30,60 \mathrm{mrad} ;(\mathrm{j})$ encircled energy for different wavelengths with the respect to the FOV angle. 


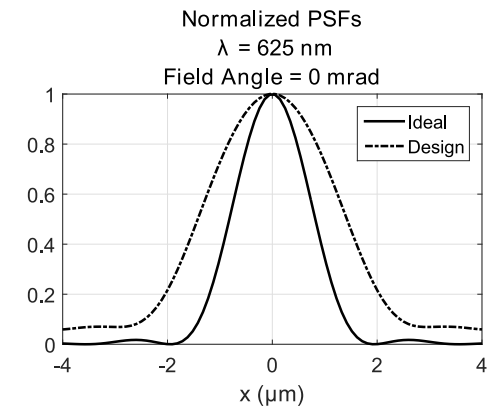

(a)

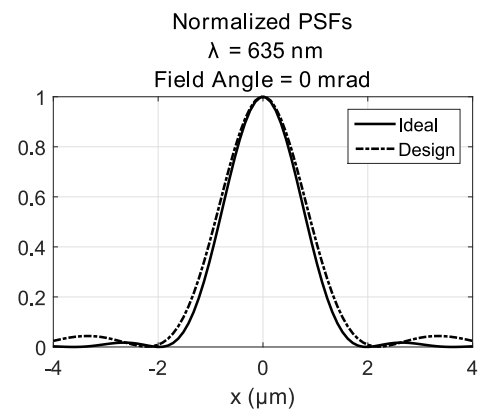

(d)

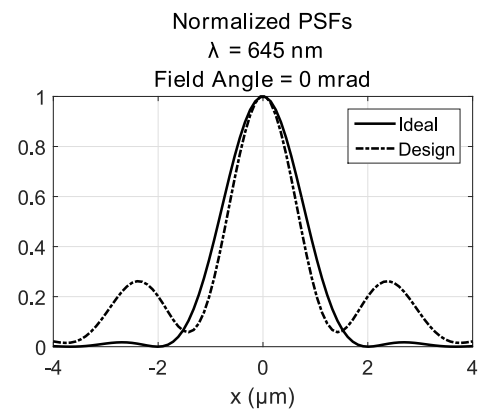

(g)

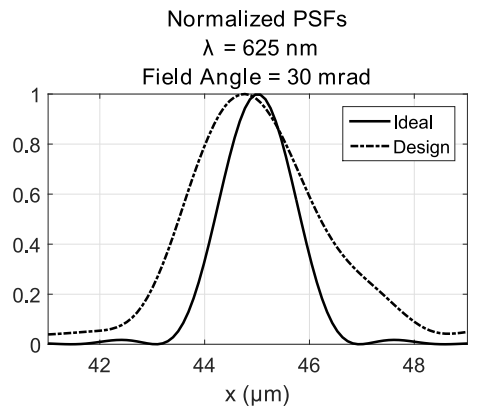

(b)

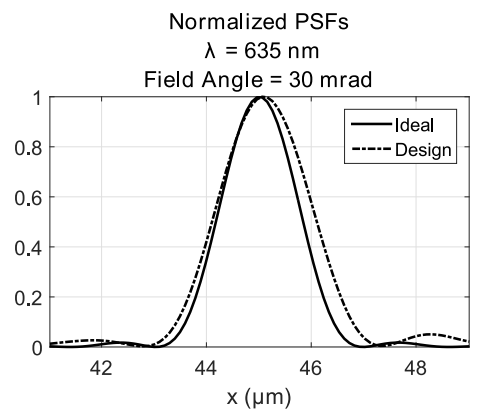

(e)

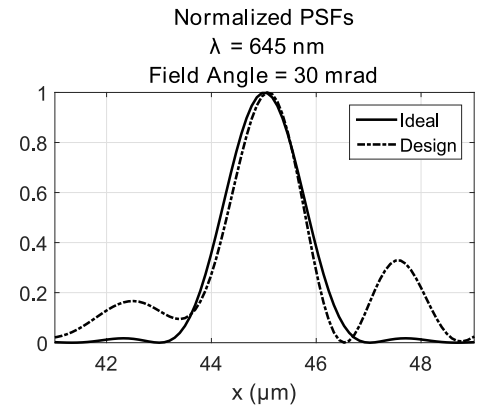

(h)

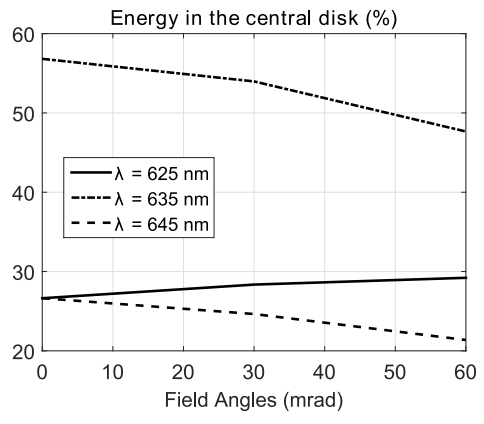

(j)

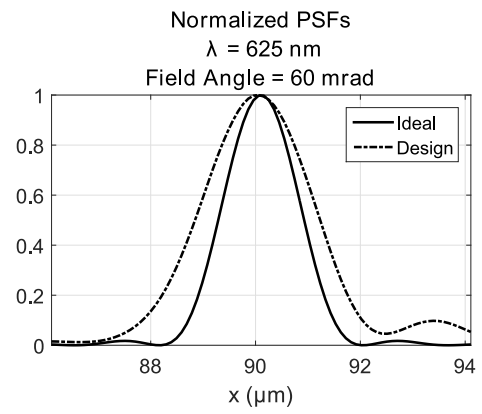

(c)

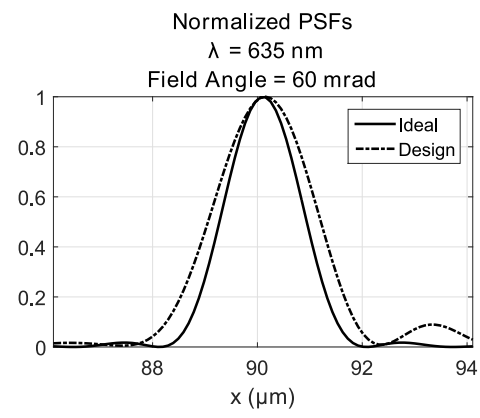

(f)

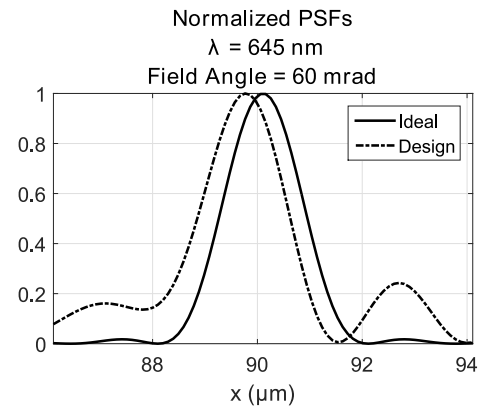

(i)

Figure 5. Focal plane intensity distributions, for different wavelengths and FOV angles, for the dielectric resonator metasurface lens realized with MS2: (a,b,c) wavelength $\lambda=625 \mathrm{~nm}, \mathrm{FOV}=0,30,60 \mathrm{mrad}$; (d,e,f) wavelength $\lambda=635 \mathrm{~nm}, \mathrm{FOV}=0,30,60 \mathrm{mrad}$; (g,h,i) wavelength $\lambda=645 \mathrm{~nm}$, FOV $=0,30,60 \mathrm{mrad}$; (j) encircled energy for different wavelengths with the respect to the FOV angle. 


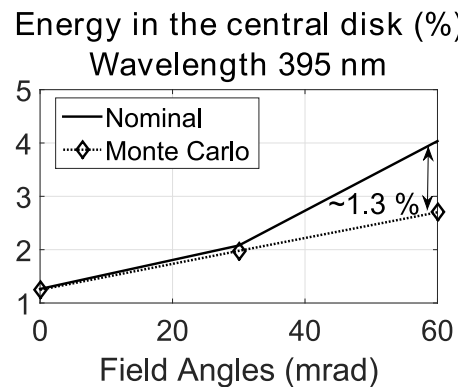

(a)

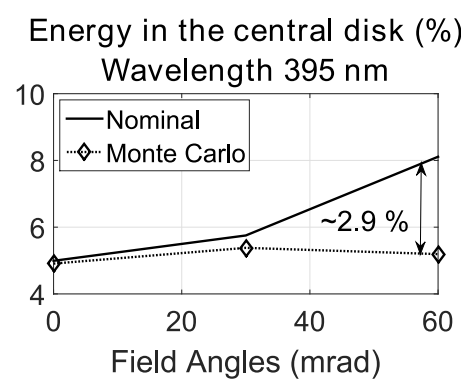

(d)

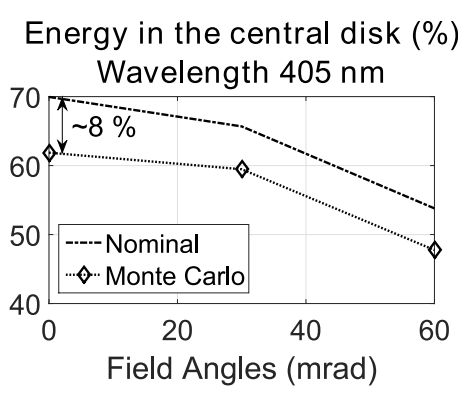

(b)

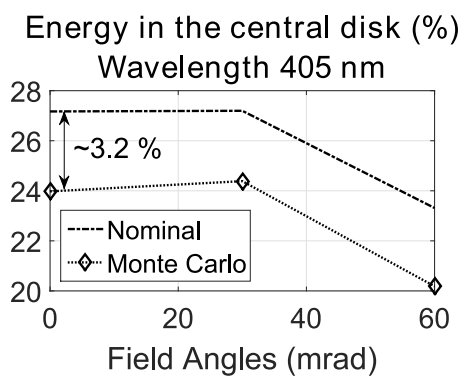

(e)

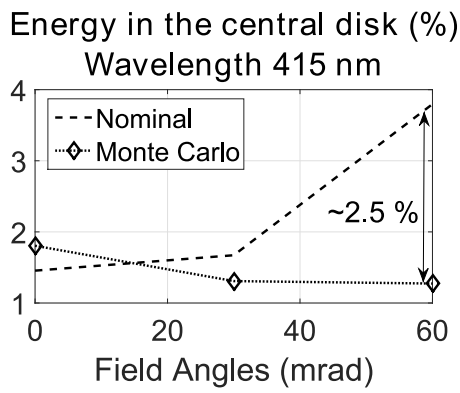

(c)

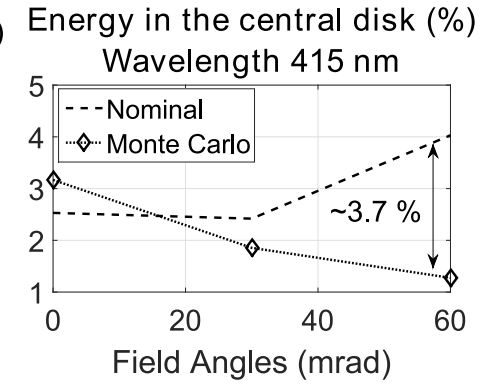

(f)

Figure 6. Per cent energy in the central disk for nominal designs vs. the average behaviour obtained with a Monte Carlo analysis (manufacturing tolerances: $\sigma^{2}=5 \mathrm{~nm}$ ), for different wavelengths: a), b), c) nominal design derived from a theoretical phase distribution: a) $395 \mathrm{~nm}$, b) $405 \mathrm{~nm}$, c) $415 \mathrm{~nm}$; d), e), f) nominal design derived from the robust optimization procedure using MS1 (same design as figure 4 d) $395 \mathrm{~nm}$, e) $405 \mathrm{~nm}, \mathrm{f}$ ) $415 \mathrm{~nm}$. The arrows in each plot show the maximum per cent variation between the nominal design and the Monte Carlo average behaviours for each wavelength. 